\title{
Secondary Intestinal Subclocation to Actinic Enteritis: Case
}

\section{Report}

\author{
Perez Castilla $A^{1 *}$, Penailillo $\mathrm{P}^{1}$, Campana $\mathrm{G}^{1}$, Oksenberg $\mathrm{D}^{2}$ and \\ Etchart $\mathbf{M}^{3}$ \\ ${ }^{1}$ Department of Digestive Surgery, Indisa Clinic, Chile \\ 2Department of Gastroenterology, Clinica Indisa, Chile \\ ${ }^{3}$ Department of Pathological Anatomy, Indisa Clinic, Chile
}

*Corresponding author: Perez Castilla A, Department of Digestive Surgery, Clinica Indisa, University Andres Bello, Santa Maria 1810 Providencia, Santiago, Chile, Tel: 56954111154; Email: investigacionindisa@gmail.com

\begin{abstract}
Introduction: Actinic enteritis is a complication derived from radiotherapy in the treatment of cancer. Almost all patients (60-90\%) undergoing radiation in the abdomen, pelvis or rectum will present with signs of acute enteritis. Complications can be acute or chronic. Surgical treatment is reserved for persistente cases or Complications.

Objective: To known the indication and the role of surgery in patients with intestinal obstruction secondary to actinic enteritis. Observing with images the macro and microscopic damage caused by actinic enteritis.

Case Report: Female 38 years, 2015 Cervical cancer treated with chemotherapy, radiotherapy and brachytherapy. 2 years since beginning treatment, It begins with pain and abdominal distensión. CT study and colonoscopy confirm subocclusion in the distal ileum secondary to actinic enteritis. Due to the persistence of symptoms, resection and subsequent reconstitution of the compromised area (ilium stenosis at $5 \mathrm{~cm}$ ileocecal valve) is performed. Pathological analysis of the resected sample is performed.
\end{abstract}

Keywords: Actinic enteritis; Brachytherapy; Stenosis; Fistula formation; Colonoscopy biopsy

\section{Introduction}

In 1887, the first case of actinic enteritis induced by radiation was published, 2 years after Wihelm Roentgen discovered the radiation [1]. Actinic enteritis is a complication derived from radiotherapy in the treatment of cancer. Almost all patients (60-90\%) undergoing radiation in the abdomen, pelvis or rectum will present with signs of acute enteritis. It is classified as acute and chronic enteritis. Acute enteritis corresponds to manifestations occurring less than 3 months after exposure. It is due to the direct effects of radiation on the intestinal mucosa. Chronic enteritis results from a transmural disease of the intestine, related to a progressive endarteritis obliterans, with subsequent ischemia and fibrosis of the same that can appear between 2 months and up to 30 years after the end of treatment [2-6].

The management of chronic actinic enteritis is continuously changing. The medical treatment of symptomatic support is the most frequently used initially. 


\section{International Journal of Surgery \& Surgical Techniques}

Surgical treatment is reserved for persistente cases or Complications such as obstruction, perforation, fistula or hemorrhage. Perioperative morbidity ranges between 30 $50 \%$ with a mortality of $10-15 \%$ [7]. At present There are still controversies regarding This topic, when is the right time and the type of surgery to be performed. It is currently assumed that the management must be individual [8-10].

\section{Case Report}

Patient of 38 years, with a history of cervical cancer diagnosed in 2015 and treated with 6 sessions of chemotherapy, 30 sessions of radiotherapy and brachytherapy. Since December of 2017, she has had colic abdominal pain associated with diarrhea. Due to the recurrence of the condition, diferente studies are performed, infection is confirmad by Clortridium Difficile, is managed with oral vancomycin. Colonoscopy is performed (Figures $1 \& 2$ ) that confirms partial stenosis of the terminal ileum, with active perimetral ulcerations of probable actinic origen. Colonoscopy biopsy result confirms actinic enteritis. A complete finding was made with abdominal CT and pelvis concluding concentric parietal thickening of small bowel loops, mainly distal ileum. New and repeated symptomatic episodes, patient was hospitalized and laparoscopic surgery was indicated. The intraoperative finding was a great adherent process of the right colon and ileum distal to the abdominal wall. Stenosis area is identified in the ileum at $5 \mathrm{~cm}$ of ileocecal valve. Resection of compromised area and reconstitution with ileo-ascending anastomosis is performed.

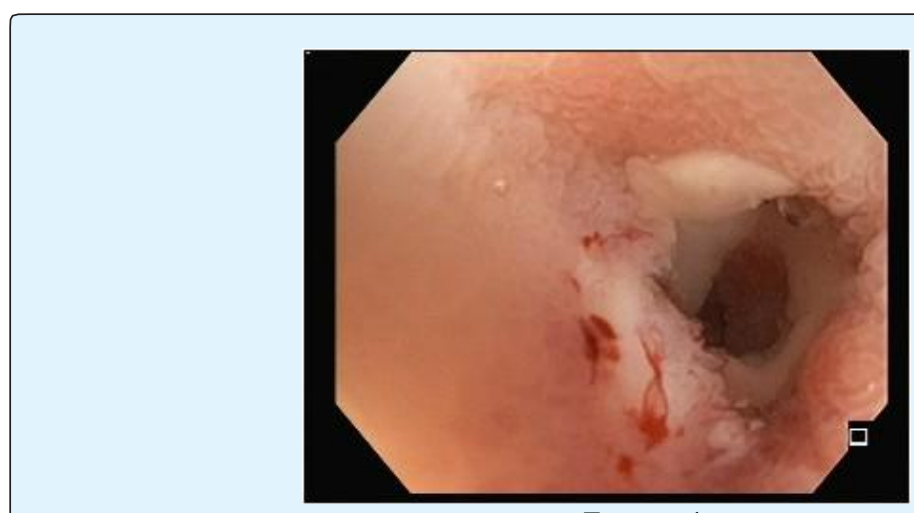

Figure 1

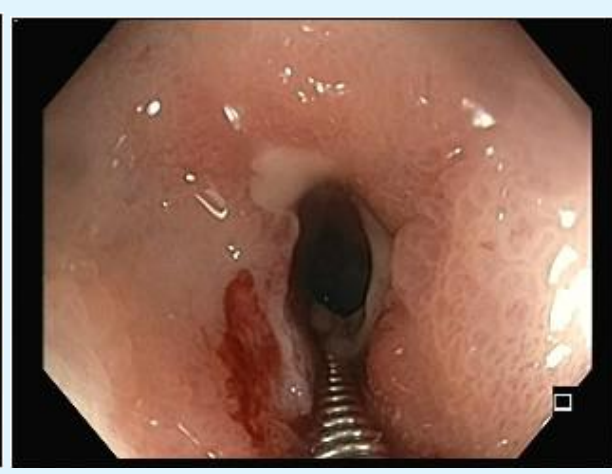

Figure 2

Figures $1 \& 2$ : Active circumferential ulceration, with fibrin all its perimeter, and which determines a lumen stenosis of about 6-7 $\mathrm{mm}$.

The pathological analysis mentions the macroscopic aspects of the lesion (Figures 3-6) observing an $18 \mathrm{~cm}$ surgical pieces with semiopaque and congestive serous, 4 $\mathrm{cm}$ ileocecal valve area of tight, infundibular annular stenosis with slight prestenotic dilatation and diffuse thickening of the ileal wall. The microscopic analysis (PHOTO 7 and 8) can be observed obliterative arteritis, dense hyaline bands and local inflammatory phenomena.

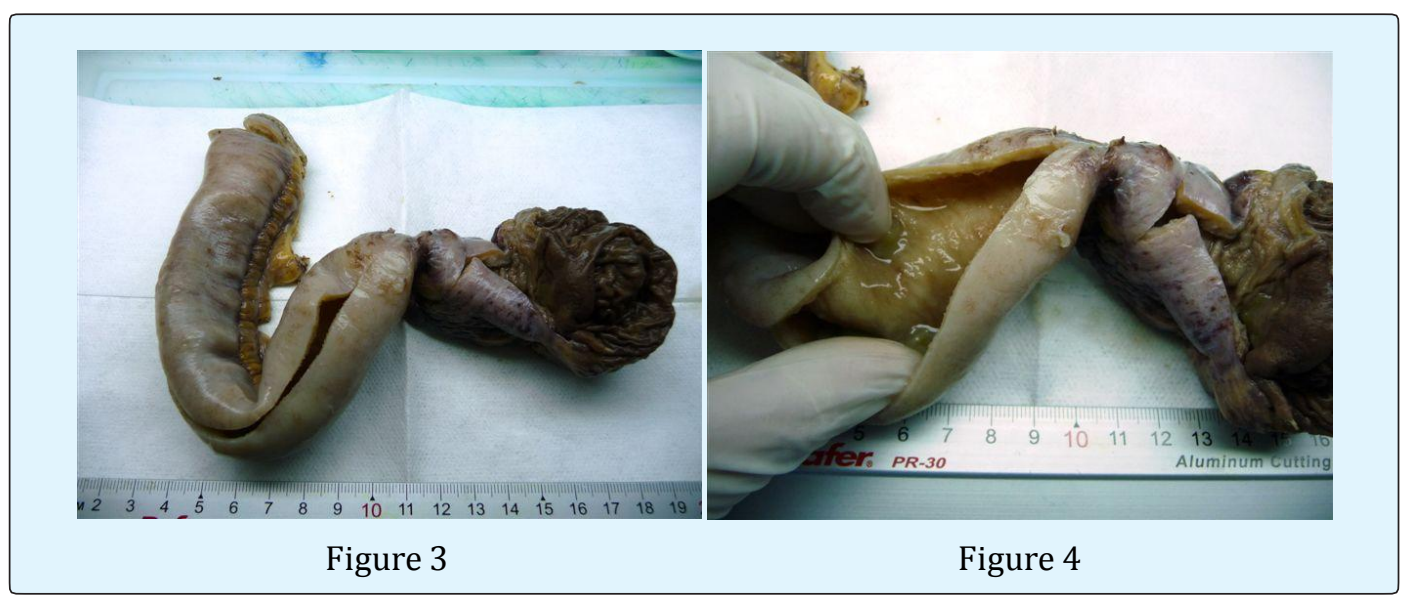

Perez Castilla A. Secondary Intestinal Subclocation to Actinic Enteritis: Case Report. Int J Surg Surgical Tech 2018, 2(3): 000127. 


\section{International Journal of Surgery \& Surgical Techniques}

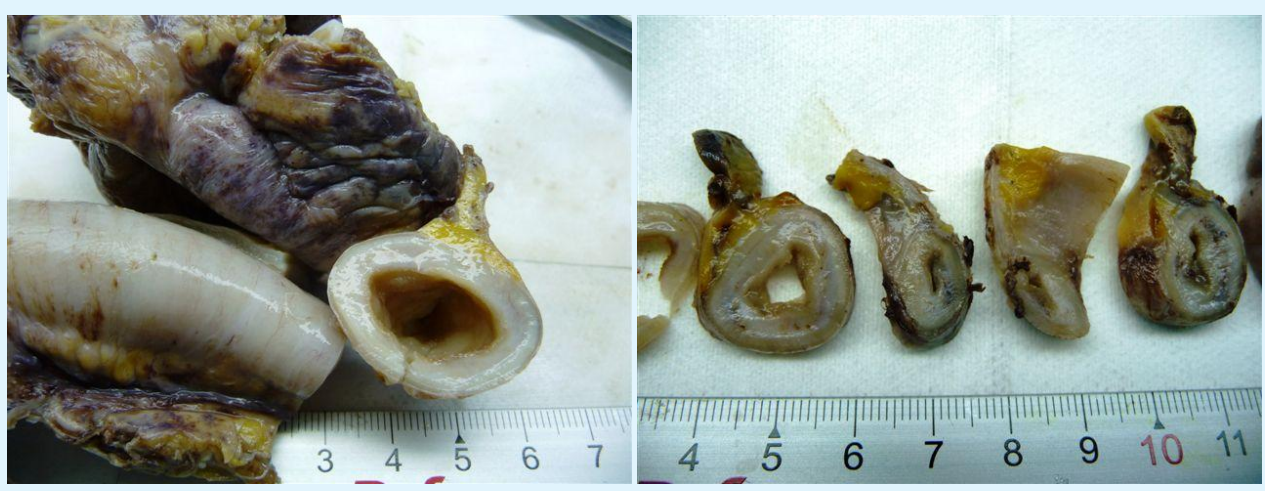

Figure 5

Figure 6

Figures 3-6: Macroscopic analysis of the segment of the small intestine (distal ileum) with signs of actinic enteritis and tight infundibular stenosis area (diameter $5-6 \mathrm{~mm}$ ). Moderate dilatation of the pre-stenotic ileal segment. Ciego de arquitectura conserved, appendix celca with phenomena of atrophy.

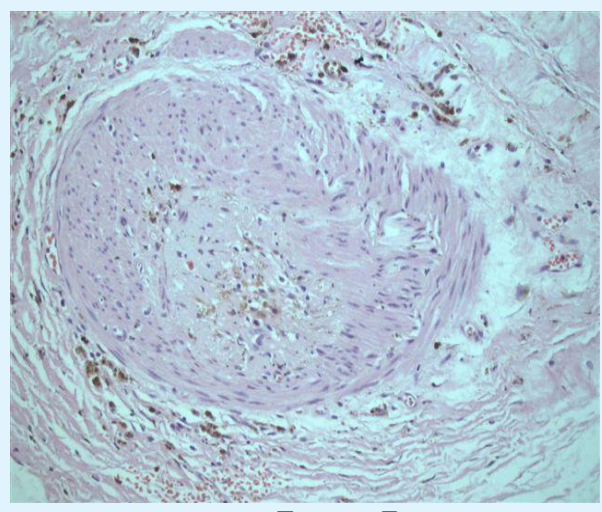

Figure 7

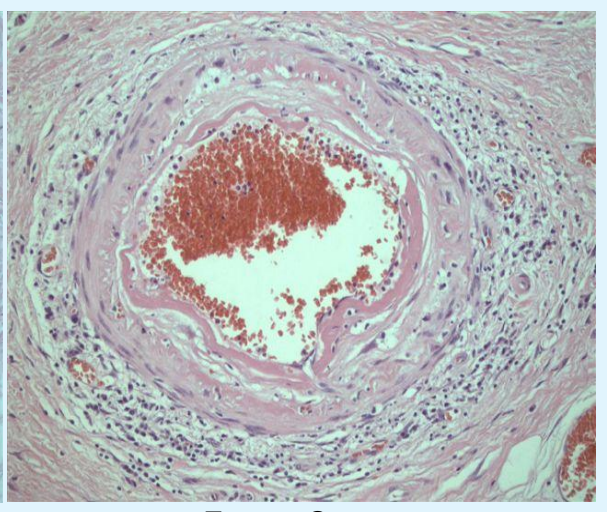

Figure 8

Figures 7 \& 8: Microscopic analysis shows erosions and superficial ulcers at the level of the stenosis zone, thickening and hyalinization of vascular walls (obliterative arteriopathy) in small mesenteric branches and moderate atypia of mesenchymal stromal cells of predominantly submucosal and reactive / degenerative nature.

\section{Discussion}

Actinic enteritis is a relevant issue in patients receiving radiotherapy for some type of cancer involving the abdomen and pelvis [11]. Such as the cervical uterine cancer that our patient presented. The proportion of patients with enteritis due to chronic radiation varies from $0.5 \%$ to $25 \%$ [12]. It is documented that the damage caused by radiation-induced enteritis is in the intestinal mucosa, due to its high rate of cell turnover $[13,14]$. Thus within the small intestine, it has been described that the duodenum and the terminal ileum are at greater risk of radiation damage because they are more susceptible to receiving more doses than mobile parts [15].
Actinic enteritis can be acute or chronic; The acute form is manifested by diarrhea and abdominal pain during or shortly after a course of radiation therapy, with resolution of symptoms often within 3 months [2,3]. Up to $90 \%$ of patients who receive pelvic radiation develop a permanent change in their defecation habits [16]. Chronic actinic enteritis develops between 6 months and 15 years after radiotherapy [17] although there are reports of up to 30 years later [4]. This chronic form is characterized by progressive endarteritis obliterans with fibrosis Exaggerated submucosa and may manifest as: malabsorption, stenosis, fistula formation, local abscesses, perforation and bleeding [12]. 


\section{International Journal of Surgery \& Surgical Techniques}

The risk factors that aggravate the presentation of the disease are related to the alteration of the microvasculature, such as diabetes mellitus, hypertension, smoking, inflammatory bowel disease, as well as radiotherapy doses and use in conjunction with chemotherapy or brachytherapy [12]. Surgery is the last therapeutic resource, with the exception of Complications (perforation, obstruction, drainage of abscesses, fistulas, infection of wounds). Less than $2 \%$ of patients who received abdominal or pelvic radiation, will require surgical intervention [9]. The timing and choice of surgical techniques remain controversial, but management must be individualized.

\section{References}

1. Walsh D (1897) Deep tissue traumatism from roentgen ray exposure. BMJ 2(1909):272-274.

2. Shadad AK, Sullivan FJ, Martin JD, Egan LJ (2013) Gastrointestinal radiation injury symptoms risk factors and mechanisms. World J Gastroenterol 19(2): 185-198.

3. Andreyev J (2005) Gastrointestinal complications of pelvic radiotherapy: are they of any importance? Gut 54(8): 1051-1054.

4. Kontouras J, Zavos C (2008) Recent advances in the management of radiation colitis. World J Gastroenterol 14(48): 7289-7301.

5. Dirksen PK, Matolo NM, Trelford JD (1977) Complications following operation in the previously irradiated abdominopelvic cavity. Am Surg 43(4): 234-241.

6. Kinsella TJ, Bloomer WD (1980) Tolerance of the intestine to radiation therapy. Surg Gynecol Obstet 151 (2): 273-284.

7. Allendorf JDF, Whelan RL (1998) Radiation injury to the small and large bowel. In: Cameron jl, ed $6^{\text {th }}$ (Edn.), current surgical therapy, St Louis Mosby, pp: 206-210.
8. Galland RB, Spencer J (1987) natural history and surgical management of radiation enteritis. Br J Surg 74(8): 742-747.

9. Galland RB, Spencer J (1986) surgical management of radiation enteritis. surgery 99 (2): 133-139.

10. Wellwood JM, Jackson BT (1973) The intestinal complications of radiotherapy. Br J Surg 60 (10): 814818.

11. Andreyev HJ (2007) Gastrointestinal problems after pelvic radiotherapy: the past, the present and the future. Clin Oncol (R Coll Radiol) 19(10): 790-799.

12. Webb GJ, Brooke R, De Silva AN (2013) Chronic radiation enteritis and malnutrition. J Dig Dis 14(7): 350-357.

13. Quastler H (1956) The nature of intestinal radiation death. Radiat Res 4(4): 303-320.

14. MacNaughton WK (2000) Review article: new insights into the pathogenesis of radiation-induced intestinal dysfunction. Aliment Pharmacol Ther 14(5): 523-528.

15. Shadad AK, Sullivan FJ, Martin JD, Egan LJ (2013) Gastrointestinal radiation injury symptoms risk factors and mechanisms. World J Gastroenterol 19(2): 185-198.

16. Olopade FA, Norman A, Blake P, Dearnaley DP, Harrington KJ, et al. (2005) A modified inflammatory Bowel Disease questionnaire and the Vaizey Incontinenc questionnaire are simple ways to identify patients with significant gastrointestinal symptoms after pelvic radiotherapy. Br J Cancer 92(9): 16631670.

17. Libotte F, Autier P, Delmelle M, Gozy M, Pector JC, et al. (1995) Survival of patients with radiation enteritis of the small and the large intestine. Acta Chir Belg 95(4): 190-194. 